\title{
Osseointegration of nanohydroxyapatite- or nano- calcium silicate-incorporated polyetheretherketone bioactive composites in vivo
}

This article was published in the following Dove Press journal:

International Journal of Nanomedicine

14 November 2016

Number of times this article has been viewed

\author{
Rui Ma ${ }^{1,2}$ \\ Zhifeng Yu' \\ Songchao Tang ${ }^{3}$ \\ Yongkang $\mathrm{Pan}^{3}$ \\ Jie $\mathrm{Wei}^{3}$ \\ Tingting Tang' \\ 'Shanghai Key Laboratory of \\ Orthopedic Implants, Department of \\ Orthopedic Surgery, Shanghai Ninth \\ People's Hospital, Shanghai Jiao Tong \\ University School of Medicine, \\ Shanghai, People's Republic of China; \\ ${ }^{2}$ Department of Orthopedic Surgery, \\ The Second Affiliated Hospital of \\ Xi'an Jiaotong University, Xi'an, \\ Shanxi Province, People's Republic of \\ China; ${ }^{3}$ Key Laboratory for Ultrafine \\ Materials of Ministry of Education, \\ School of Materials Science and \\ Engineering, East China University \\ of Science and Technology, Shanghai, \\ People's Republic of China
}

Correspondence: Tingting Tang Shanghai Key Laboratory of Orthopedic Implants, Department of Orthopedic Surgery, Shanghai Ninth People's Hospital, Shanghai Jiao Tong University School of Medicine, 639 Zhizaoju Road, Shanghai 2000 I I, People's Republic of China

Tel +86 2I 2327 | I 33

$\mathrm{Fax}+862163137020$

Email tingtingtang@hotmail.com

Jie Wei

Key Laboratory for Ultrafine Materials of Ministry of Education, School of Materials Science and Engineering, East China University of Science and Technology, I 30 Meilong Road, Shanghai 200237,

People's Republic of China

Email jiewei7860@sina.com

\begin{abstract}
Polyetheretherketone (PEEK) exhibits appropriate biomechanical strength as well as good biocompatibility and stable chemical properties but lacks bioactivity and cannot achieve highly efficient osseointegration after implantation. Incorporating bioceramics into the PEEK matrix is a feasible approach for improving its bioactivity. In this study, nanohydroxyapatite (n-HA) and nano-calcium silicate (n-CS) were separately incorporated into PEEK to prepare n-HA/PEEK and n-CS/PEEK biocomposites, respectively, using a compounding and injectionmolding technique, and the in vitro degradation characteristics were evaluated. Discs with a diameter of $8 \mathrm{~mm}$ were inserted in $8 \mathrm{~mm}$ full-thickness cranial defects in rabbits for 4 and 8 weeks, and implantation of pure PEEK was used as the control. Three-dimensional microcomputed tomography, histological analysis, fluorescence microscopy of new bone formation, and scanning electron microscopy were used to evaluate the osseointegration performance at the bone/ implant interface. The results of the in vitro degradation study demonstrated that degradation of $\mathrm{n}$-CS on the surface of $\mathrm{n}-\mathrm{CS} / \mathrm{PEEK}$ could release $\mathrm{Ca}$ and $\mathrm{Si}$ ions and form a porous structure. In vivo tests revealed that both $n-C S / P E E K$ and $n-H A / P E E K$ promoted osseointegration at the bone/implant interface compared to PEEK, and n-CS/PEEK exhibited higher bone contact ratio and more new bone formation compared with those of n-HA/PEEK, implying that n-CS/PEEK possessed a stronger ability to promote osseointegration. These two PEEK biocomposites are promising materials for the preparation of orthopedic or craniofacial implants.
\end{abstract}

Keywords: polyetheretherketone, composite, osseointegration, hydroxyapatite, calcium silicate

\section{Introduction}

As a polycyclic aromatic thermoplastic, polyetheretherketone (PEEK) is widely used in orthopedic clinics. PEEK has an adjustable mechanical property that is similar to that of human cortical bone, and this can mitigate concerns over the risks of bone resorption caused by stress shielding as a result of elasticity mismatch between metallic implants and human bones. ${ }^{1}$ Moreover, PEEK exhibits stable chemical properties, good biocompatibility and natural radiolucency. ${ }^{2,3}$ However, PEEK is a bioinert material, ${ }^{1}$ and its implantation in a living body could result in encapsulation of fibrous tissues that isolates the implant from the surrounding bone tissues. ${ }^{4}$ In a study of intervertebral fusion, the osseointegration efficiency of PEEK-Cage was lower than that of Ti-Cage. ${ }^{5}$ Webster et $\mathrm{l}^{6}$ implanted machined PEEK in a skull defect and found the bone contact ratio was only approximately $8 \%$. In a clinical study, the combined use of bone morphogenetic protein-2 (BMP-2) with PEEK increased the fusion rate (FR) to $90.6 \%,{ }^{7}$ while the FR of pure PEEK was only $70.6 \% .{ }^{8}$ However, the use of BMP2 submit your manuscript Dovepress AY通口

http://dx,doi.org/10.2147/IJN.S11528\% cC) and incorporate the Creative Commons Attribution - Non Commercial (unported, v3.0) License (http://creativecommons.org/licenses/by-nc/3.0/). By accessing the work you hereby accept the Terms. Non-commercial uses of the work are permitted without any further permission from Dove Medical Press Limited, provided the work is properly attributed. For permission for commercial use of this work, please see paragraphs 4.2 and 5 of our Terms (https://www.dovepress.com/terms.php). 
is too expensive and may induce ectopic bone formation, radiculitis, and soft tissue swelling. ${ }^{9}$

Modifying PEEK to improve its bioactivity can reduce the rate of implantation failure caused by poor osseointegration and avoid secondary surgeries, reducing the economic burden of the patients. Currently, two strategies are available for improving the bioactivity of PEEK including composite preparation (incorporating bioactive materials into PEEK) and surface treatment/coating with physical/chemical methods. ${ }^{2}$ Among these strategies, impregnating bioactive materials into PEEK has become an attractive approach for improving the bioactivity of PEEK while maintaining its mechanical properties. ${ }^{10}$ Ceramics with bone-bonding ability are defined as bioactive ceramics, such as hydroxyapatite (HA), calcium silicate (CS), bioglasses, etc. Synthetic HA material is biocompatible and bioactive and is clinically used as an important bone substitute. ${ }^{11}$ Some studies have proved that CS is biocompatible, biodegradable, and bioactive, with the ability to stimulate proliferation and osteogenic differentiation of osteoblasts, ${ }^{12-16}$ and CS has been shown to have higher bioactivity than calcium phosphate materials. ${ }^{17-19}$ Abu Bakar et $\mathrm{al}^{20}$ prepared 20 volume \% (vol\%) HA-reinforced PEEK composite (HA/PEEK) with a porosity of $60 \%$ and pore size ranging from 300 to $600 \mathrm{~mm}$ using a leaching of particulate technique employing a suitable pore-forming agent, and these materials were implanted into the distal metaphyseal femur of pigs to evaluate the biological responses and tissue ingrowth of the material. Histological studies revealed the presence of fibrovascular tissue within the pores after 6 weeks and mature bone formation after 16 weeks, indicating that the HA/PEEK composite exhibited favorable osseointegration. Ma et $\mathrm{al}^{21}$ successfully prepared a HA/PEEK composite via an in situ synthetic process and implanted it into femurs of Sprague Dawley rats. In this case, the new bone tissues surrounding the composite implants grew faster with a higher HA content. The interface between new bones and the HA/PEEK composite was seamless after 3 months of implantation, suggesting that reliable biological fixation can be achieved. Kim et $\mathrm{al}^{22}$ fabricated CS-reinforced PEEK composite (CS/PEEK) with 0-50 vol\% CS and soaked specimens in simulated body fluid with pure PEEK as the control. Except for pure PEEK, all of the CS-containing composites promoted apatite formation on their surfaces, and the time required for the induction of apatite formation on the composite surfaces decreased with increasing CS content. Considering both mechanical properties and bioactivity, these authors selected $20 \mathrm{vol} \% \mathrm{CS} / \mathrm{PEEK}$ as a promising implant material.

Although most studies employ microsized particles to impregnate PEEK, some nanosized materials have been used to prepare nanocomposites to improve the biomechanical properties and biological activity. ${ }^{23,24}$ In previous studies, ${ }^{25,26}$ our research group used a compounding and injection-molding technique to separately incorporate nanohydroxyapatite (n-HA) and nano-calcium silicate (n-CS) into PEEK to prepare n-HA/PEEK and n-CS/PEEK composites, respectively. The optimal incorporating ratio for both n-HA and n-CS was 40 weight $\%$ (wt $\%$ ), and both $n-H A / P E E K$ and $n-C S /$ PEEK exhibited good bioactivity, which was confirmed by a simulated body fluid soaking test and in vitro cell tests. In the current study, a skull defect model in rabbits was used to evaluate the osseointegration of the two PEEK composites in vivo.

\section{Materials and methods Preparation of materials}

Our previous results ${ }^{25,26}$ indicated that elastic modulus and compressive strength of the composite increased with increasing $\mathrm{n}-\mathrm{HA}$ or $\mathrm{n}-\mathrm{CS}$ content from 0 to $40 \mathrm{wt} \%$, while the highest $\mathrm{n}-\mathrm{HA}$ or $\mathrm{n}-\mathrm{CS}$ content $(60 \mathrm{wt} \%$ ) yielded a lower elastic modulus and compressive strength, so the concentration of n-HA or $\mathrm{n}-\mathrm{CS}$ in PEEK composite in this study was determined as 40 wt $\%$. The $40 \mathrm{wt} \% \mathrm{n}-\mathrm{HA} / \mathrm{PEEK}$ and $40 \mathrm{wt} \% \mathrm{n}-\mathrm{CS} / \mathrm{PEEK}$ composites were fabricated using a compounding and injectionmolding technique according to our previously reported procedure, ${ }^{25,26}$ and the pure PEEK sample was prepared using an injection-molding technique. Briefly, the PEEK and $\mathrm{n}-\mathrm{HA} / \mathrm{n}-\mathrm{CS}$ powders were compounded in a high-speed ball mill (QM-3B, T-Bota Scietech Instruments \& Equipment Ltd., Nanjing, People's Republic of China) at a mixing speed of $500 \mathrm{rpm}$ for 1 hour; the mixtures were then dried at $150^{\circ} \mathrm{C}$ for 24 hours; and the samples were produced using an injection-molding machine (Battenfeld BA-300/050CD, Awans, Belgium) at an injecting and molding temperature of $380^{\circ} \mathrm{C}$. All the samples were cut into discs with a thickness of $2 \mathrm{~mm}$ and a diameter of $8 \mathrm{~mm}$ and cleaned with deionized water for 2 hours in an ultrasonic oscillator (B3500S-MT, Branson, Danbury, CT, USA) followed by drying at $37^{\circ} \mathrm{C}$ overnight and sterilization with ethylene oxide.

\section{In vitro degradation test}

The pure PEEK, n-HA/PEEK, and n-CS/PEEK samples were soaked in a trihydroxymethyl aminomethane-hydrochloric acid (Tris-HCl) buffer solution ( $\mathrm{pH}=7.40)$ with a surface areato-volume ratio of $0.1 \mathrm{~cm}^{-1}$ at a shaking speed of $120 \mathrm{rpm}$ at $37^{\circ} \mathrm{C}$ for $1,3,7,14,21$, and 28 days. At each time point, the samples were removed and the concentrations of calcium (Ca) and silicon ( $\mathrm{Si}$ ) ions in the soaked fluids measured 
using inductively coupled plasma atomic emission spectroscopy (ICP-AES; Varian, Palo Alto, CA, USA). The surface morphologies of the samples prior to immersion and after 28 days of immersion were observed using scanning electron microscopy (SEM; S-4800, Hitachi, Tokyo, Japan).

\section{Animal model}

Mature female New Zealand White rabbits were used as the experimental animals, and a skull defect was selected as the bone defect model. ${ }^{27}$ The operative procedures and animal care were performed according to the Principles of Shanghai 9th People's Hospital on Animal Experimentation. Ethical approval for undertaking the animal experiment was obtained from the institutional ethics board of the Shanghai Ninth People's Hospital. Anesthesia was performed by intramuscular injection of $2 \%$ xylazine $(12 \mathrm{mg} / \mathrm{kg}$ ) and ketamine $(80 \mathrm{mg} / \mathrm{kg})$. The skull was shaved and washed, and the animals were immobilized in the ventral position. After the area disinfected with iodine, a longitudinal incision was made down to the periosteum from the nasal bone to the occipital protuberance at the left parietal bone. Then, a midline incision was made in the periosteum, and the periosteum was undermined and lifted off the parietal skull. The defect was drilled from the outside plate, diplane, and internal plate in the middle of the left parietal bone with a trephine (Med-X Research Institute, Shanghai Jiaotong University, Shanghai, People's Republic of China) such that the outer diameter was $8.0 \mathrm{~mm}$ (Figure 1A). Care was taken to make a full thickness defect without damaging the underlying dura. After insertion of the implants (Figure 1B), the periosteum, subcutaneous tissue, and skin were sutured layer by layer (Figure 1C). To reduce perioperative infection, the rabbits received an intramuscular injection of penicillin (100,000 U per rabbit) within 3 days after surgery. After 4 weeks of implantation, the implants with the surrounding tissues were retrieved and cut into small samples $(2 \times 2 \mathrm{~cm})$. The samples were scanned with microcomputed tomography (Micro-CT; $\mu$ CT80, SCANCO, Bern, Switzerland) with a resolution of $21 \mu \mathrm{m}$, radiographs were prepared, and then the CTvol software package was used to reconstruct the three-dimensional photos. Figure 1D shows the reconstructed photo of the skull defect after 4 weeks, and the image indicates that the skull defect model in the rabbit was successfully established.

\section{Groups and implant retrieval}

Thirty mature female New Zealand White rabbits weighting $2.4 \pm 0.2 \mathrm{~kg}$ were divided into three groups (ie, PEEK,
A
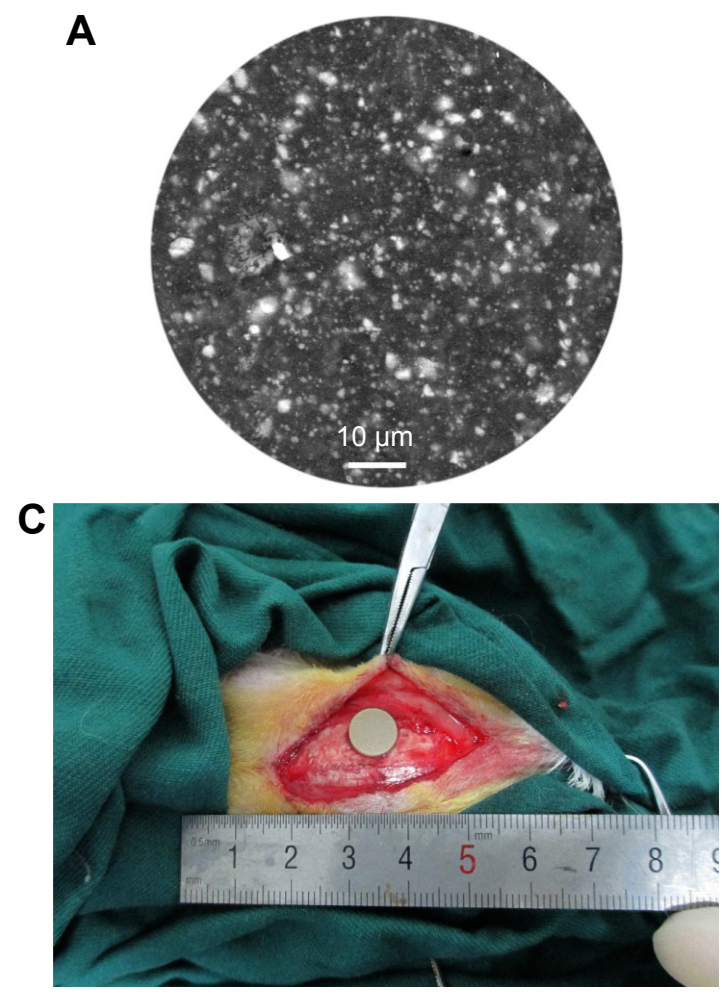

B
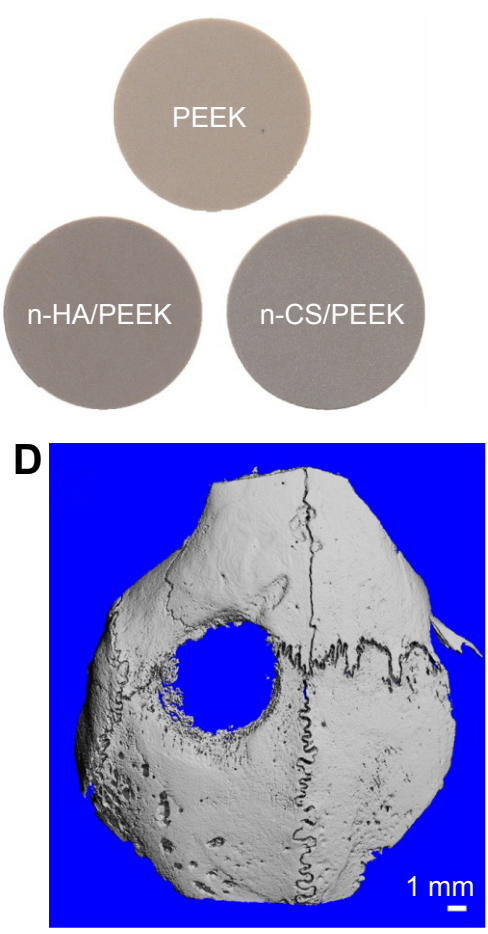

Figure I Implantation of composites in rabbit skull defect.

Notes: (A) Representative SEM image showed that n-HA was distributed evenly in the matrix of PEEK. (B) Implanted samples. (C) Implants were inserted into the parietal bone defect. (D) The reconstructed image of the skull defect after 4 weeks of implantation.

Abbreviations: PEEK, polyetheretherketone; n-HA, nanohydroxyapatite; n-CS, nano-calcium silicate; SEM, scanning electron microscopy. 
n-HA/PEEK, and n-CS/PEEK groups, Figure 1B). Another three rabbits without implants served as blank controls, which were evaluated by Micro-CT only. The rabbits were intramuscularly injected with alizarin red solution $(30 \mathrm{mg} / \mathrm{kg})$, calcein solution $(20 \mathrm{mg} / \mathrm{kg})$, and alizarin red solution $(30 \mathrm{mg} / \mathrm{kg})$ at 3 weeks, 2 weeks, and 1 week prior being sacrificed, respectively. After being sacrificed, the left parietal bones were retrieved, and the soft tissues were removed. Then, the samples were soaked in 75\%/25\% ethanol/water for 1 week; this was followed by dehydration in an ethanol gradient at ethanol/water volume fractions of $80 \%, 95 \%$, and $100 \%$ ( 2 days for each gradient).

\section{Micro-CT analysis}

The retrieved parietal bones containing the implants were cut into $2 \times 2 \mathrm{~cm}$ sizes with the implant in the center. The specimens were scanned and reconstructed at $21 \mu \mathrm{m}$ (resolution) using a micro-CT to observe the bone/implant interface.

\section{Preparation of histological section}

The specimens were placed into the embedded device containing the methylmethacrylate (MMA) monomer (9100, Technovit, Wehrheim, Germany) and then stored at $4^{\circ} \mathrm{C}$. After 1 week, the MMA monomer was discarded. Colloidal MMA was added and stored at room temperature until solidified. The embedded fragments containing the tissue specimens were collected and cut into $150 \mu \mathrm{m}$ sections with a microtome (SP 1600, Leica, Wetzlar, Germany). Then, the sections were adhered to organic glass slides and compressed for 24 hours. The thickness of the section was polished to $50 \mu \mathrm{m}$ using P300, P800, and P1200 abrasive paper followed by burnishing with flannelette and abradum to $20-30 \mu \mathrm{m}$.

\section{Histological evaluation}

At least three sections of each implant were stained with picric acid/fuchsine. The histological sections were soaked in $1 \%$ formic acid for 3 minutes, rinsed with running water for 5 minutes, and dried. Prior to rinsing with running water for 5 minutes again, the histological sections were soaked in $20 \%$ methanol for 2 hours. Then, the picric acid/fuchsin staining was performed. The histological sections were preheated at $60^{\circ} \mathrm{C}$, stained with Stevenol blue for 5-15 minutes, rinsed with distilled water and dried, stained with the VG staining solution for 3-8 minutes, cleaned with $100 \%$ ethanol, and dried. A light microscope (Leica Microsystems AG, Wetzlar, Germany) was used for histological evaluation. Finally, the bone contact ratio between the implant surface and the surrounding bone were quantitatively analyzed using the
Bioquant Osteo II (Bioquant Image Analysis Corporation, Nashville, TN, USA) image analysis software.

\section{Fluorescence microscopy of new bone formation}

The fluorescence of the new bone formation labeled with alizarin red and calcein was visualized using confocal laser scanning microscopy (CLSM, Leica TCS SP2, Leica Microsystems, Heidelberg, Germany).

\section{SEM observations}

The specimens were sputter-coated with gold for 60 seconds, and the interface between the implant and the bone was observed using SEM in the backscattered electron mode (S-4800, Hitachi). The accelerating voltage used was $10.0 \mathrm{kV}$.

\section{Statistical analysis}

All the data are presented as the means \pm standard deviations. All the statistical analyses were performed using the SPSS software (version 13.0; IBM Corporation, Armonk, NY, USA). The statistical significance between different groups was analyzed using a two-way analysis of variance (ANOVA) test, and multiple comparisons were performed using the least significant difference test. $P$-values $<0.05$ were considered significant, and $P$-values $<0.01$ were considered highly significant.

\section{Results}

\section{In vitro degradation test}

Figure 2 shows the results of the in vitro degradation test. The SEM images of then-CS/PEEK surface after soaking for 28 days indicated that a porous structure had formed after the n-CS particles dissolved (Figure 2A). In contrast, no obvious change was observed on the surface of PEEK and n-HA/PEEK. Pure PEEK released neither $\mathrm{Ca}$ ions nor $\mathrm{Si}$ ions. n-HA/PEEK released $\mathrm{Ca}$ ions at a high rate within 3 days and at a low rate after 3 days (Figure $2 \mathrm{~B}$ and $\mathrm{C}$ ). In contrast, n-CS/PEEK continuously released $\mathrm{Ca}$ and $\mathrm{Si}$ ions for up to 21 days. Within 3 days, $n-C S / P E E K$ quickly released a mass of $\mathrm{Ca}$ and $\mathrm{Si}$ ions, but after 3 days the release quantity and rate of $\mathrm{Ca}$ and $\mathrm{Si}$ ions decreased substantially. From days 21 to 28, the dissolution curves for both the $\mathrm{Ca}$ and $\mathrm{Si}$ ions reached a plateau, implying that little to no $\mathrm{Ca}$ or $\mathrm{Si}$ ions were released from the composite.

\section{Micro-CT analysis}

The $3 \mathrm{D}$ reconstructed images of micro-CT are shown in Figure 3. The PEEK was radiolucent, and n-HA and n-CS 
A
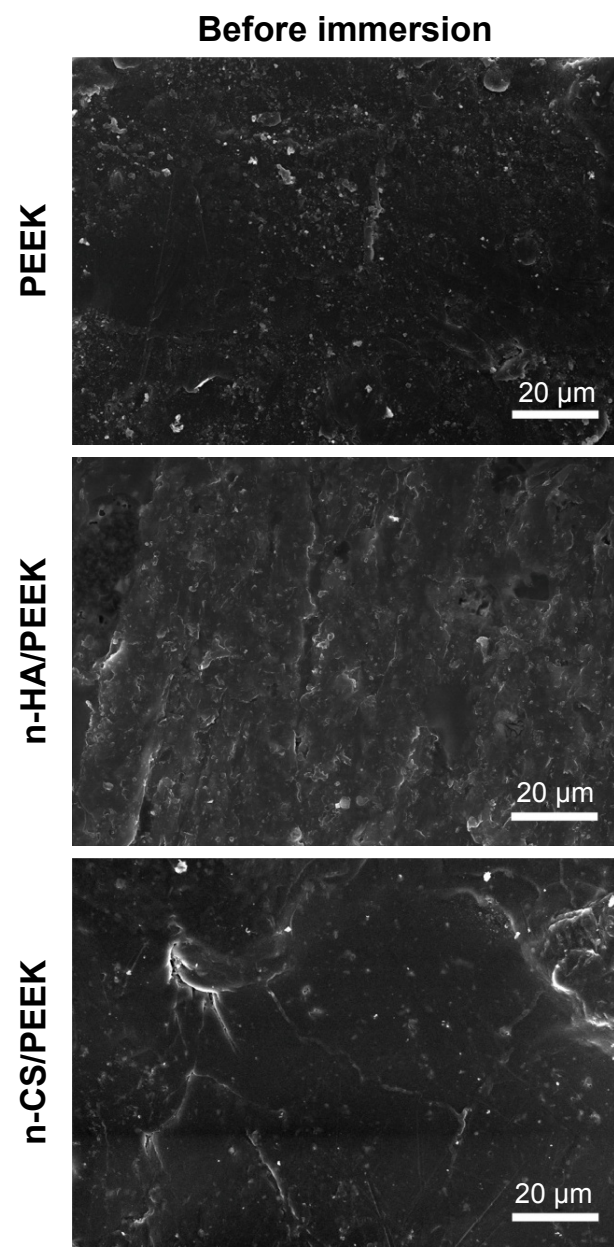

B

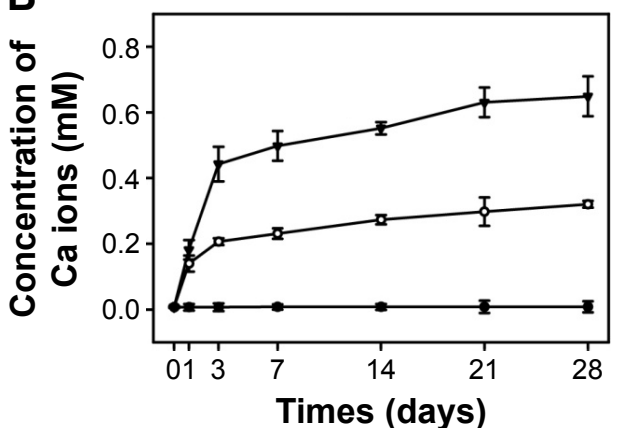

After immersion
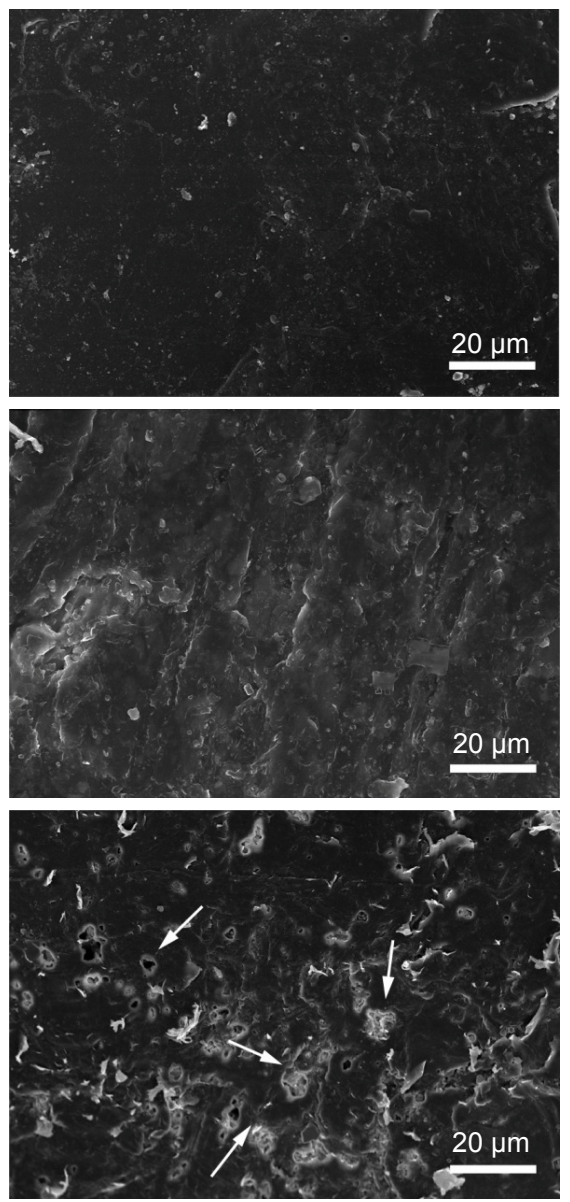

C

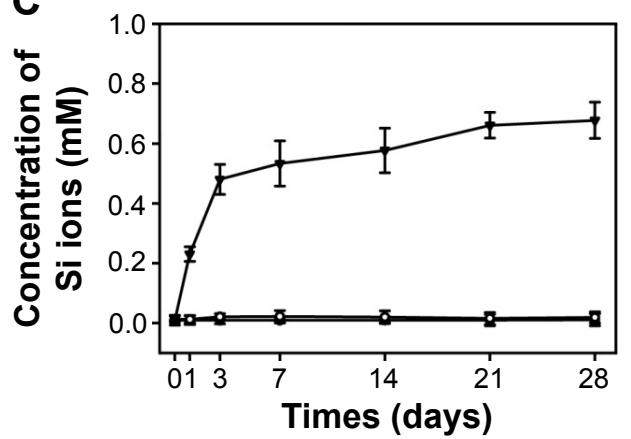

$\rightarrow$ PEEK $\multimap \mathrm{n}-\mathrm{HA} / \mathrm{PEEK} \rightarrow \mathrm{n}-\mathrm{CS} / \mathrm{PEEK}$

Figure 2 In vitro degradation.

Notes: (A) SEM images of the samples before and after immersion in a Tris- $\mathrm{HCl}$ solution for 28 days. The arrows indicated that a porous structure had formed after the $\mathrm{n}$-CS particles dissolved. (B) Concentration of $\mathrm{Ca}$ ions in the soaked solution. (C) Concentration of Si ions in the soaked solution.

Abbreviations: PEEK, polyetheretherketone; n-HA, nanohydroxyapatite; $\mathrm{n}-\mathrm{CS}$, nano-calcium silicate; SEM, scanning electron microscopy; Tris-HCl, trihydroxymethyl aminomethane-hydrochloric acid.

could be imaged. The lack of gaps between the inorganic particles and the surrounding bone indicated that bone contact or bone integration existed (black arrows). At 4 weeks, the surface of PEEK was relatively smooth and bone ongrowth was limited. However, a small portion of bone contact was observed around n-HA/PEEK and n-CS/PEEK. At 8 weeks, bone growth around PEEK was still limited, and a majority of the bone contact had been formed on the n-HA/PEEK surface even though a slight gap was observed around it. In contrast, the interface around n-CS/PEEK 

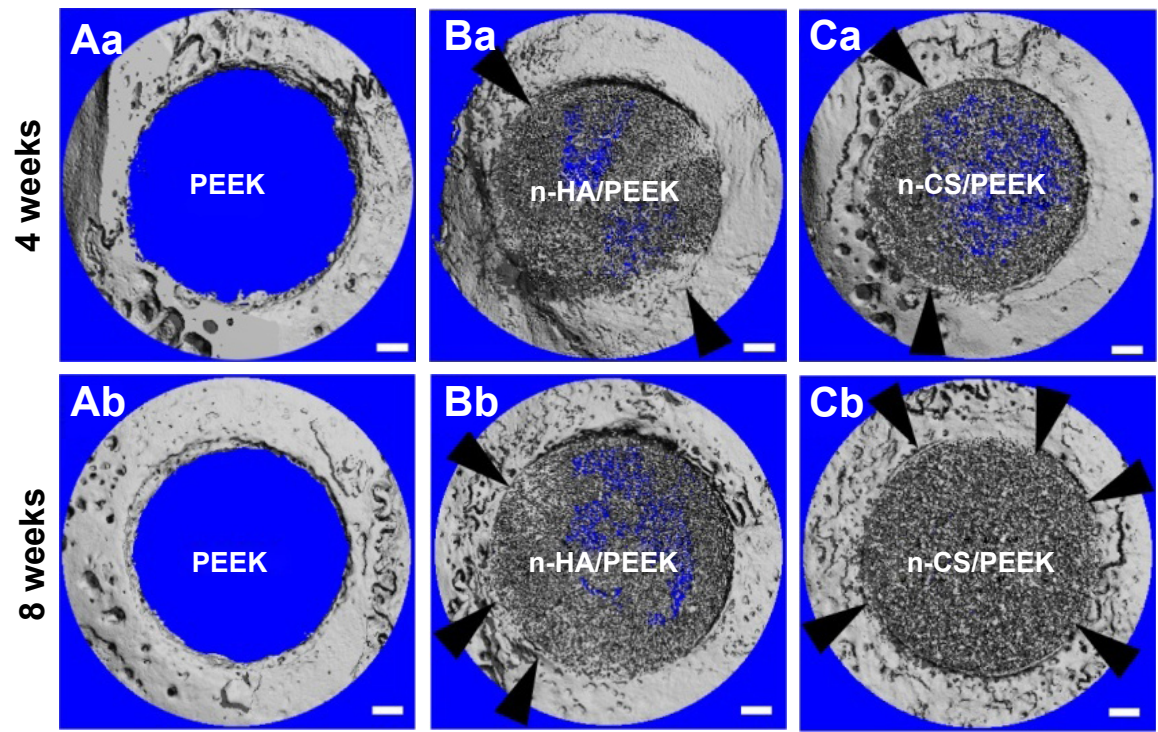

Figure 3 Representative images of the 3D reconstruction of the cranial defect in rabbits using micro-CT at 4 and 8 weeks after implantation. Notes: The black arrows indicate bone contact. The scale bar is I $\mu \mathrm{m}$.

Abbreviations: PEEK, polyetheretherketone; n-HA, nanohydroxyapatite; n-CS, nano-calcium silicate; 3D, three-dimensional; Micro-CT, microcomputed tomography.

was seamless, indicating the successful osseointegration of n-CS/PEEK.

\section{Histological evaluation}

Figure 4 shows the histological results of osseointegration after implantation. At 4 weeks, no direct bone contact was observed, but a layer of fibrous connective tissue (indicated by the black arrows in Figure 4) was observed around PEEK. Bone contact (indicated by the white arrows in Figure 4A) was found around n-HA/PEEK, and more obvious new bone tissues were in close contact with the surface of n-CS/PEEK. At 8 weeks, the fibrous connective tissue around PEEK still existed, and obvious bone tissues had grown on the surface of n-HA/PEEK. In comparison, the surface of n-CS/PEEK was covered by abundant bone tissue. The bone contact ratio of n-HA/PEEK and n-CS/PEEK were higher than that of PEEK $(P<0.01)$. In addition, n-CS/PEEK exhibited a higher bone contact ratio than that of n-HA/ PEEK $(P<0.05)$.

\section{Fluorescence microscopy of new bone formation}

The new bone formation around the implant surface was fluorescently labeled and observed by CLSM, and the results are shown in Figure 5. Red fluorescence was due to alizarin labeling, and yellow-green fluorescence was due to calcein labeling. Three fluorescence bands were observed from the material side to the bone side as follows: red fluorescence, yellow-green fluorescence, and red fluorescence. The width between two fluorescence labels (interlabeled width) represents the amount of new bone formation within each time interval. At 4 weeks, the three fluorescence labels around the PEEK surface overlapped with each other (Figure 5A), indicating that the new bone formation around PEEK was limited. Both qualitative and quantitative results indicated that the interlabeled widths around $\mathrm{n}-\mathrm{HA} / \mathrm{PEEK}$ and $\mathrm{n}$-CS/PEEK were larger than that of PEEK $(P<0.01)$, which indicated new bone had formed around these two composites. At 8 weeks, significantly more new bone formation was observed around n-HA/PEEK and n-CS/PEEK compared with that around PEEK $(P<0.01)$. Comparisons between $\mathrm{n}$-HA/PEEK and n-CS/PEEK further indicated that more new bone was formed around n-CS/PEEK compared with that observed for n-HA/PEEK at 4 weeks $(P<0.01)$ and 8 weeks $(P<0.05)$ (Figure 5B).

\section{SEM observations}

The bone/implant interface was observed using SEM, and the results are shown in Figure 6. The bone tissues bonded closely with the surface of n-HA/PEEK (Figure 6B) and n-CS/PEEK (Figure 6C) with no obvious gap, which demonstrated that stable fixation had been achieved. However, an obvious gap between PEEK and the surrounding bone was observed (Figure 6A), indicating that the integration at the PEEK surface was poor. 

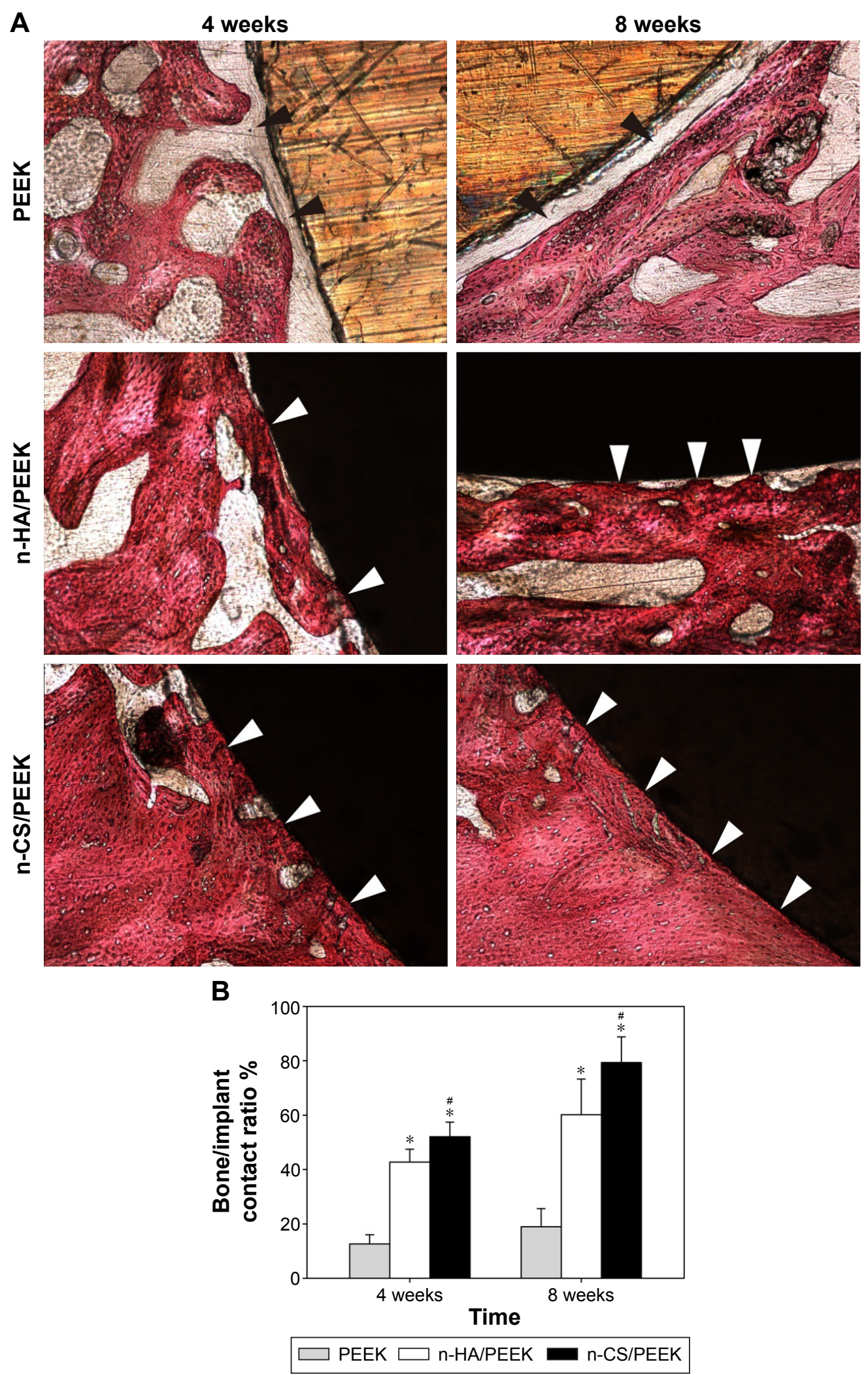

Figure 4 Histological analysis of osseointegration 4 and 8 weeks after implantation.

Notes: (A) Histological micrographs. The black arrows indicate fibrous connective tissue, and the white arrows indicate bone contact. (B) Comparison of percentage of

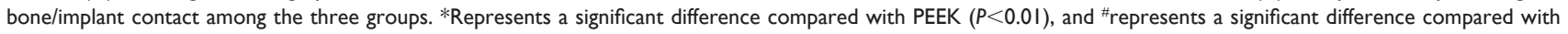
n-HA/PEEK $(P<0.05)$.

Abbreviations: PEEK, polyetheretherketone; n-HA, nano-hydroxyapatite; n-CS, nano-calcium silicate. 
A
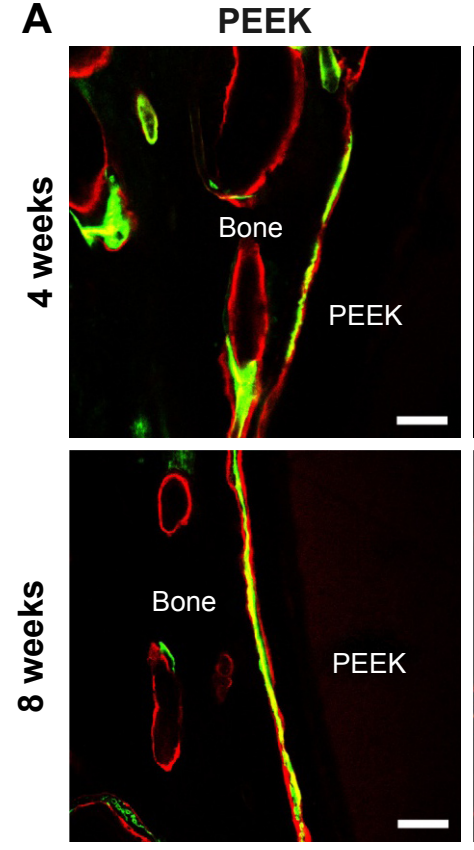

B
n-HA/PEEK
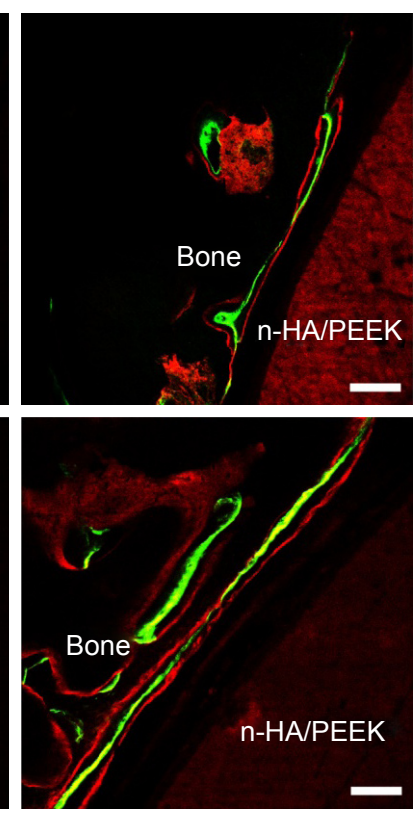

n-CS/PEEK
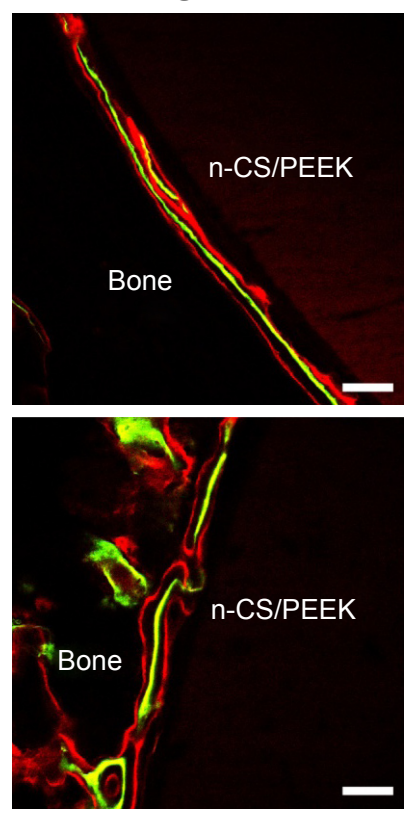

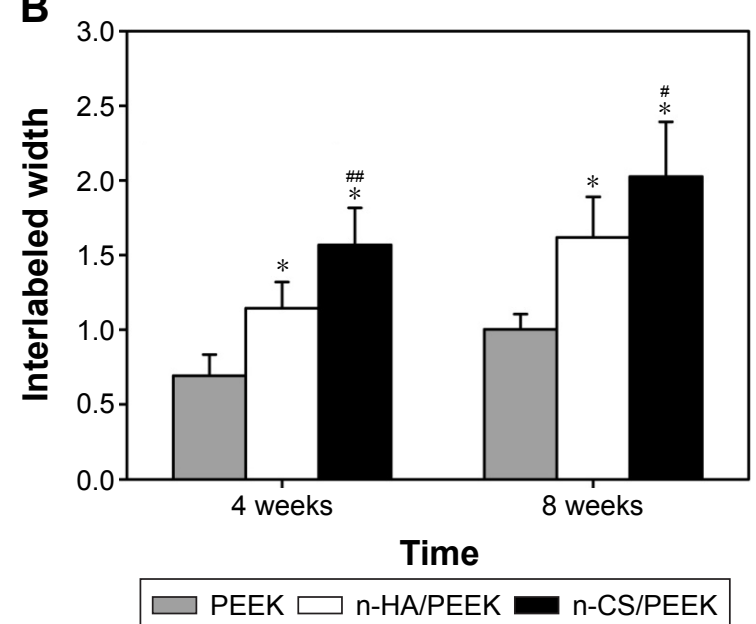

Figure 5 Fluorescence microscopy of new bone formation labeled by alizarin red and calcein after 4 and 8 weeks.

Notes: (A) Representative CLSM images. The red fluorescence is due to alizarin red labeling, and the yellow-green fluorescence is due to by calcein labeling. The scale bar is $100 \mu \mathrm{m}$. (B) Quantitative analysis of new bone formation. *Represents a significant difference compared to PEEK $(P<0.0 \mathrm{I})$, and ${ }^{\#}$ represents a significant difference compared with n-HA/PEEK $\left({ }^{\#}<<0.05,{ }^{\# P}<0.01\right)$.

Abbreviations: PEEK, polyetheretherketone; n-HA, nanohydroxyapatite; n-CS, nano-calcium silicate; CLSM, confocal laser scanning microscopy.

\section{Discussion}

Osseointegration is the direct contact between the bone and implant, without the presence of fibrous connective tissue in-between, when observed under an optical microscope. ${ }^{28}$ A bioactive implant must have the ability to stimulate a biological response to achieve osseointegration at the bone/ implant interface. ${ }^{29,30}$ Only an integrated bone/implant interface results in long-term stability of the structure and function of the interface. ${ }^{31,32}$ For successful osseointegration, the biomaterials should exhibit bioactive interactions with osteoblasts. In general, an apatite layer that forms on the material surface could mimic the environment of the recipient bone to promote adhesion and differentiation of osteogenic cells. ${ }^{11,33}$ Incorporation with bioactive particles or surface modification can endow inert materials with the ability to stimulate osteoblast growth.

In the current study, we separately incorporated two nanoscale bioceramics (n-HA and n-CS) into PEEK to prepare PEEK biocomposites, and then, these biocomposites were implanted into cranial defects of rabbits to evaluate the osseointegration at the bone/implant interface. One study was found reporting on osseointegration of carbon 

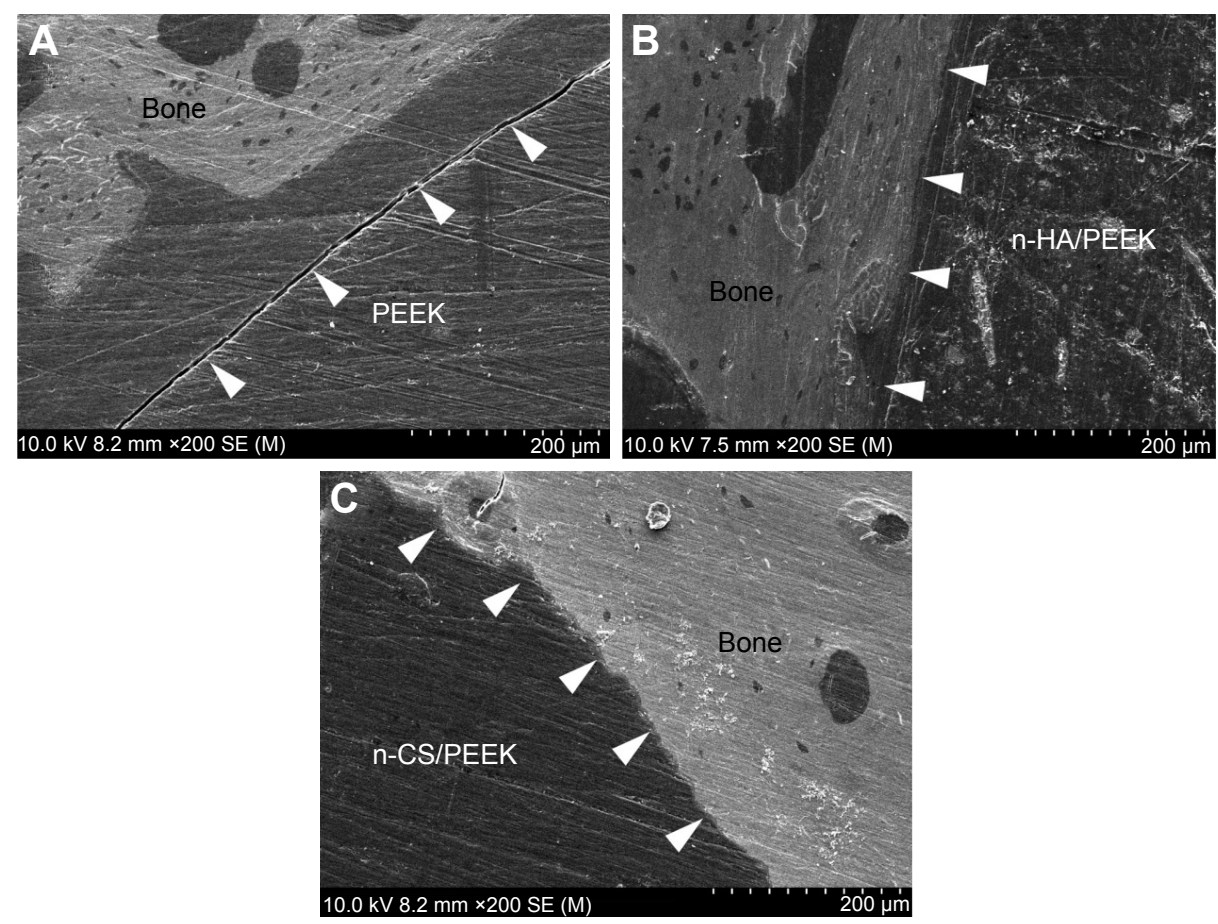

Figure 6 SEM images of the bone/implant interfaces after 8 weeks.

Notes: The white arrows indicate the bone/implant interfaces around (A) PEEK, (B) n-HA/PEEK, and (C) n-CS/PEEK.

Abbreviations: PEEK, polyetheretherketone; n-HA, nanohydroxyapatite; n-CS, nano-calcium silicate; SEM, scanning electron microscopy.

fiber-reinforced polyetheretherketone-nanohydroxyapatite biocomposite (PEEK/CF/n-HA) in the mandibles of beagle dogs,${ }^{34}$ but no study has reported the in vivo osseointegration of $\mathrm{n}-\mathrm{CS} / \mathrm{PEEK}$ composite in any animal model. Micro-CT is often applied to calculate bone apposition around an implant, analyze the three-dimensional structure of the scaffold, and observe the bone ingrowth of the scaffold. ${ }^{35,36}$ The $3 \mathrm{D}$ reconstructed micro-CT images indicated that the bone contact around n-HA/PEEK and n-CS/PEEK was more obvious than that around PEEK. In the histological micrograph, a large amount of bone tissues was in close contact with the n-HA/ PEEK and n-CS/PEEK surfaces and PEEK was surrounded by a layer of fibrous connective tissue. The quantitative results indicated that the bone contact ratio of n-CS/PEEK was significantly higher than that of n-HA/PEEK. The improved osteointegration around the PEEK composites may be caused by improved surface characteristics.

The surface characteristics of the material (ie, composition, topography, and hydrophilicity) are very important to osseointegration. ${ }^{37,38}$ A surface with moderate roughness and strong hydrophilicity is favorable for cell activity and osseointegration..$^{39,40}$ In an animal test, Shalabi et a ${ }^{41}$ reported that a rough surface exhibited a higher osseointegration efficiency than that of a smooth surface. In our previous study, addition of n-HA or n-CS improved the surface roughness and hydrophilicity of PEEK, which are beneficial to the adhesion, proliferation, spread, and osteogenic differentiation of osteoblasts. In addition, because nanomaterials support adhesion and differentiation of osteoblasts, ${ }^{42,43}$ the nanoscale HA on n-HA/PEEK and nanoscale CS on n-CS/ PEEK may also contribute to the improved osseointegration of the composites. Xu et al ${ }^{13}$ developed a novel carbon fiber-reinforced polyetheretherketone-nanohydroxyapatite (PEEK/CF/n-HA) ternary biocomposite with a micro-/ nanotopographical surface. Their results indicated that the micro-/nanotopographical PEEK/CF/n-HA biocomposite exhibited outstanding ability to promote the proliferation and differentiation of MG-63 cells in vitro as well as boost osseointegration between the implant and bone in vivo.

New bone formation is often labeled by alizarin red and calcein, which can combine with inorganic salts in vivo and deposit in the mineralized bone matrix. ${ }^{27}$ More new bone formation was observed around n-HA/PEEK and n-CS/ PEEK compared with that around PEEK at both the early stage (4 weeks) and late stage ( 8 weeks), and more active new bone formation was observed around n-CS/PEEK compared with that around n-HA/PEEK. To the best of our knowledge, this is the first study to demonstrate that both n-HA and n-CS incorporation could improve the in vivo osseointegration of the composite and that n-CS/PEEK exhibits a higher 
osseointegration efficiency than that of n-HA/PEEK. The better performance of n-CS/PEEK may be due to the higher concentration of $\mathrm{Ca}$ and $\mathrm{Si}$ ions released from this composite. The $\mathrm{Ca}$ and $\mathrm{Si}$ ions were beneficial for proliferation and differentiation of osteoblasts, ${ }^{19}$ and a porous structure on the composite surface permitted more new bone growth in the pores, leading to better osseointegration. In addition, CS offers improved bioactivity compared with that of calcium phosphate materials. ${ }^{18,19}$ In our previous study, ${ }^{25}$ an apatite layer was formed on the n-CS/PEEK composite, and the entire surface of this specimen was nearly covered with a thick and compact apatite layer after immersion for 28 days. We predicted that apatite would form on the surface of n-CS/ PEEK in the early implantation period, and then, the bone matrix would integrate with the apatite.

\section{Conclusion}

In this study, a rabbit model with cranial defect was used to investigate the osseointegration around two PEEK biocomposites (ie, n-HA/PEEK and n-CS/PEEK). On the basis of the results of micro-CT scans, histological studies, CLSM observations, and SEM observations, it can be seen that the incorporation of both n-HA and n-CS into PEEK materials promoted the osseointegration of implants in vivo and that $\mathrm{n}-\mathrm{CS} / \mathrm{PEEK}$ induced more active bone formation around the implants, which may be due to the $\mathrm{Ca}$ and $\mathrm{Si}$ ions being released from the composite. These results demonstrated that both n-CS/PEEK and n-HA/PEEK can be used to make bioactive implants for craniofacial or orthopedic uses.

\section{Acknowledgments}

This research was financially supported by the National Key R\&D Program (2016YFC1102100), the National Natural Science Foundation of China (31271015, 81271705, and 81572194), and the Shanghai Science and Technology Development Fund (15441902500).

\section{Disclosure}

The authors declare no conflicts of interest in this work.

\section{References}

1. Wang $\mathrm{H}, \mathrm{Xu} \mathrm{M}$, Zhang $\mathrm{W}$, et al. Mechanical and biological characteristics of diamond-like carbon coated poly aryl-ether-ether-ketone. Biomaterials. 2010;31(32):8181-8187.

2. Ma R, Tang T. Current strategies to improve the bioactivity of PEEK. Int J Mol Sci. 2014;15(4):5426-5445.

3. Kurtz SM, Devine JN. PEEK biomaterials in trauma, orthopedic, and spinal implants. Biomaterials. 2007;28(32):4845-4869.

4. Anderson JM. Biological responses to materials. Annu Rev Mater Res. 2001;31:81-110.
5. Toth JM, Wang M, Estes BT, et al. Polyetheretherketone as a biomaterial for spinal applications. Biomaterials. 2006;27(3):324-334.

6. Webster TJ, Patel AA, Rahaman MN, Sonny Bal B. Anti-infective and osteointegration properties of silicon nitride, poly(ether ether ketone), and titanium implants. Acta Biomater. 2012;8(12):4447-4454.

7. Behrbalk B, Uri O, Parks RM, Musson R, Soh RC, Boszczyk BM. Fusion and subsidence rate of stand alone anterior lumbar interbody fusion using PK cage with recombinant human bone morphogenetic protein-2. Eur Spine J. 2013;22(12):2869-2875.

8. Strube P, Hoff E, Hartwig T, Perka CF, Gross C, Putzier M. Standalone anterior versus anteroposterior lumbar interbody single-level fusion after a mean follow-up of 41 months. Spinal Disord Tech. 2012; 25(7):362-369.

9. Shimer AL, Oner FC, Vaccaro AR. Spinal reconstruction and bone morphogenetic proteins: open questions. Injury. 2009;40(Suppl3): S32-S38.

10. Roeder RK, Conrad TL. Chapter 11 - Bioactive polyaryletherketone composites. In: Kurtz SM, editors. PEEK Biomaterials Handbook. Oxford, UK: William Andrew Publishing; 2012:163-179.

11. Kokubo T, Kim H-M, Kawashita M. Novel bioactive materials with different mechanical properties. Biomaterials. 2003;24(13):2161-2175.

12. Li H, Zhai W, Chang J. Effects of wollastonite on proliferation and differentiation of human bone marrow-derived stromal cells in PHBV/ wollastonite composite scaffolds. J Biomater Appl. 2009;24(3): 231-246.

13. Li H, Chang J. Fabrication and characterization of bioactive wollastonite/ PHBV composite scaffolds. Biomaterials. 2004;25(24):5473-5480.

14. Siriphannon P, Kameshima Y, Yasumori A, Okada K, Hayashi S. Influence of preparation conditions on the microstructure and bioactivity of alpha- $\left.\mathrm{CaSiO}_{3}\right)$ ceramics: formation of hydroxyapatite in simulated body fluid. J Biomed Mater Res. 2000;52(1):30-39.

15. De Aza PN, Luklinska ZB, Martinez A, Anseau MR, Guitian F, De Aza S. Morphological and structural study of pseudowollastonite implants in bone. J Microsc. 2000;197(Pt 1):60-67.

16. Lin K, Zhai W, Ni S, Chang J, Zeng Y, Qian W. Study of the mechanical property and in vitro biocompatibility of $\mathrm{CaSiO}_{3}$ ceramics. Ceram Int . 2005;31:323-326.

17. Kobayashi M, Nakamura T, Okada Y, et al. Bioactive bone cement: comparison of apatite and wollastonite containing glass-ceramic, hydroxyapatite, and beta-tricalcium phosphate fillers on bone-bonding strength. J Biomed Mater Res. 1998;42(2):223-237.

18. Oonishi H, Hench LL, Wilson J, et al. Quantitative comparison of bone growth behavior in granules of Bioglass, A-W glass-ceramic, and hydroxyapatite. J Biomed Mater Res. 2000;51(1):37-46.

19. Ni S, Chang J, Chou L, Zhai W. Comparison of osteoblast-like cell responses to calcium silicate and tricalcium phosphate ceramics in vitro. J Biomed Mater Res B Appl Biomater. 2007;80(1):174-183.

20. Abu Bakar MS, Cheng MHW, Tang SM, et al. Tensile properties, tension-tension fatigue and biological response of polyetheretherketonehydroxyapatite composites for load-bearing orthopedic implants. Biomaterials. 2003;24(13):2245-2250.

21. Ma R, Weng L, Bao X, Song S, Zhang Y. In vivo biocompatibility and bioactivity of in situ synthesized hydroxyapatite/polyetheretherketone composite materials. J Appl Polym Sci. 2013;127(4):2581-2587.

22. Kim IY, Sugino A, Kikuta K, Ohtsuki C, Cho SB. Bioactive composites consisting of PEEK and calcium silicate powders. J Biomater Appl. 2009;24(2):105-118.

23. Ma R, Weng L, Fang L, Luo Z, Song S. Structure and mechanical performance of in situ synthesized hydroxyapatite/polyetheretherketone nanocomposite materials. J Sol-Gel Sci Techn. 2012;62(1):52-56.

24. Li K, Yeung CY, Yeung K, Tjong C. Sintered hydroxyapatite/ polyetheretherketone nanocomposites: mechanical behavior and biocompatibility. Adv Eng Mater. 2012;14(4):B155-B165. 
25. Ma R, Tang S, Tan H, et al. Preparation, characterization, in vitro bioactivity, and cellular responses to a polyetheretherketone bioactive composite containing nanocalcium silicate for bone repair. ACS Appl Mater Interfaces. 2014;6(15):12214-12225.

26. Ma R, Tang S, Tan H, et al. Preparation, characterization, and in vitro osteoblast functions of a nano-hydroxyapatite/polyetheretherketone biocomposite as orthopedic implant material. Inter J Nanomedicine. 2014;9: 3949-3961.

27. Ruhé PQ, Kroese-Deutman HC, Wolke JG, Spauwen PH, Jansen JA. Bone inductive properties of rhBMP-2 loaded porous calcium phosphate cement implants in cranial defects in rabbits. Biomaterials. 2004;25(11): 2123-2132.

28. Coelho PG, Jimbo R. Osseointegration of metallic devices: current trends based on implant hardware design. Arch Biochem Biophys. 2014; 561:99-108

29. Hench LL, Splinter RJ, Allen W, Greenlee TK Jr. Bonding mechanisms at the interface of ceramic prosthetic materials. J Biomed Mater Res. 1971;5:117-141.

30. Ao H, Xie Y, Yang S, et al. Covalently immobilised type I collagen facilitates osteoconduction and osseointegration of titanium coated implants. J Orthop Transl. 2016;5:16-25.

31. Nishiguchi S, Nakamura T, Kobayashi M, Kim HM, Miyaji F, Kokubo T. The effect of heat treatment on bone-bonding ability of alkali-treated titanium. Biomaterials. 1999;20:491-500.

32. Kim HM, Takadama H, Kokubo T, Nishiguchi S, Nakamura T. Formation of a bioactive graded surface structure on Ti-15Mo-5Zr-3Al alloy by chemical treatment. Biomaterials. 2000;21:353-358.

33. Lee JH, Jang HL, Lee KM, et al. In vitro and in vivo evaluation of the bioactivity of hydroxyapatite-coated polyetheretherketone biocomposites created by cold spray technology. Acta Biomater. 2013;9(4): 6177-6187.

34. Xu A, Liu X, Gao X, Deng F, Deng Y, Wei S. Enhancement of osteogenesis on micro/nano-topographical carbon fiber-reinforced polyetheretherketone-nanohydroxyapatite biocomposite. Mater Sci Eng C Mater Biol Appl. 2015;48:592-598.
35. Jones AC, Arns CH, Sheppard AP, Hutmacher DW, Milthorpe BK, Knackstedt MA. Assessment of bone ingrowth into porous biomaterials using Micro-CT. Biomaterials. 2007;28(15):2491-2504.

36. Alt V, Lips KS, Henkenbehrens C, et al. A new animal model for implant-related infected non-unions after intramedullary fixation of the tibia in rats with fluorescent in situ hybridization of bacteria in bone infection. Bone. 2011;48(5):1146-1153.

37. Boyan BD, Hummert TW, Dean DD, Schwartz Z. Role of material surfaces in regulating bone and cartilage cell response. Biomaterials. 1996; 17(2):137-146.

38. Steigenga JT, Al-Shammari KF, Nociti FH, Misch CE, Wang HL. Dental implant design and its relationship to long-term implant success. Implant Dent. 2003;12(4):306-317.

39. Feller L, Chandran R, Khammissa RA, et al. Osseointegration: biological events in relation to characteristics of the implant surface. SADJ. 2014;69(3):112, 114-117

40. Stach RM, Kohles SS. A meta-analysis examining the clinical survivability of machined-surfaced and osseotite implants in poor-quality bone. Implant Dent. 2003;12(1):87-96.

41. Shalabi MM, Wolke JG, Jansen JA. The effects of implant surface roughness and surgical technique on implant fixation in an in vitro model. Clin Oral Implants Res. 2006;17(2):172-178.

42. Zhu X, Eibl O, Scheideler L, Geis-Gerstorfer J. Characterization of nano hydroxyapatite/collagen surfaces and cellular behaviors. J Biomed Mater Res A. 2006;79(1):114-127.

43. Kumar A, Young C, Farina J, Witzlc A, Marks ED. Novel nanocomposite biomaterial to differentiate bone marrow mesenchymal stem cells to the osteogenic lineage for bone restoration. J Orthop Transl. 2015;3(3): $105-113$.
International Journal of Nanomedicine

\section{Publish your work in this journal}

The International Journal of Nanomedicine is an international, peerreviewed journal focusing on the application of nanotechnology in diagnostics, therapeutics, and drug delivery systems throughout the biomedical field. This journal is indexed on PubMed Central, MedLine, CAS, SciSearch $\AA$, Current Contents $₫ /$ Clinical Medicine,

\section{Dovepress}

Journal Citation Reports/Science Edition, EMBase, Scopus and the Elsevier Bibliographic databases. The manuscript management system is completely online and includes a very quick and fair peer-review system, which is all easy to use. Visit http://www.dovepress.com/ testimonials.php to read real quotes from published authors. 